\title{
Demonstration of record-high 352-Gbps terahertz wired transmission over hollow-core fiber at $325 \mathrm{GHz}$
}

\author{
Min ZHU ${ }^{1,2}$, Jiao ZHANG ${ }^{1,2}$, Jianjun $\mathrm{YU}^{2,3^{*}} \&$ Xiaohu YOU ${ }^{1,2}$ \\ ${ }^{1}$ National Mobile Communications Research Laboratory, Southeast University, Nanjing 210096, China; \\ ${ }^{2}$ Purple Mountain Laboratories, Nanjing 211111, China; \\ ${ }^{3}$ School of Information Science and Technology, Fudan University, Shanghai 200433, China
}

Received 20 October 2021/Accepted 2 November 2021/Published online 19 December 2021

Citation Zhu M, Zhang J, Yu J J, et al. Demonstration of record-high 352-Gbps terahertz wired transmission over hollow-core fiber at $325 \mathrm{GHz}$. Sci China Inf Sci, 2022, 65(2): 127301, https://doi.org/10.1007/s11432-021-3361-5

Terahertz $(\mathrm{THz})$ waves, extending from 0.1 to $10 \mathrm{THz}$, are attracting extensive attention in the interdisciplinary fields of electronics and photonics. The $\mathrm{THz}$ band offers a larger available bandwidth, which can meet the Tbps transmission capacity for $6 \mathrm{G}$ wireless communication. Moreover, it could enable high-resolution sensing and imaging to implement Internet of Things (IoT) for trillion communicating devices, owing to the short wavelength compared to the microwave system [1]. However, the big obstacle of free-space $\mathrm{THz}$ wireless link is limited by the high atmospheric attenuation, and hence it just can be applicable to the line-of-sight transmission scenario. $\mathrm{THz}$ wired link based on specific fiber working at $\mathrm{THz}$ band has attractive features including light weight, low attenuation, flexibility, electromagnetic compatibility, and the capacity to support non-line-of-sight transmission [2]. Hence, the seamless integration transmission of $\mathrm{THz}$ wireless and wired links can leverage both approaches to expand $\mathrm{THz}$ practical application scenarios, which has great potential for future information and communication technologies.

Research review. The photonic-aided THz-wave communication scheme has been investigated to offer a variety of advantages over conventional all-electronic approaches. These advantages include wideband tunability of the wireless carrier frequency, large bandwidth, less harmonics, and seamless integration with optical transmission. Additionally, advanced multi-dimension multiplexing techniques in the optical domain such as wavelength/polarization division multiplexing (WDM/PDM), high-order modulation, can be used to realize broadband wireless transmission at line rates of 100 Gbps and beyond [3-5]. However, the photonicsassisted systems are still limited by the lower transmit power and the higher atmospheric attenuation in practical transmission. Integrating optical fibers, the transparent opticalTHz-optical link is demonstrated to provide line-rates up to 240 and 190 Gbps over 5 and $115 \mathrm{~m}$ wireless distances at $230 \mathrm{GHz}$. Transparency is enabled by a plasmonic modulator which can map a $\mathrm{THz}$ signal directly onto an optical carrier in an optical fiber [6]. Uncompressed high-definition $4 \mathrm{~K}$ video (8 Gbps) transmission at $330 \mathrm{GHz}$ has been successfully transmitted, as a proof of concept that the terahertz wireless link can directly interface with the terahertz optical fiber wired link [2]. The THz fiber is composed of an air-core region surrounded by a thin and low refractive index expanded polytetrafluoroethylene cladding layer, and the photonic-crystal waveguide is effectively designed for coupling $\mathrm{THz}$ fiber and wireless link. In W-band at $95 \mathrm{GHz}$, the 128 Gbps transmission has been demonstrated over two parallel spans of 1.5-m dielectric-coated metallic hollow fiber (DMHF) based on space division multiplexing [7]. The hollow-core fiber for efficient $\mathrm{THz}$ signal delivery could provide lower dispersion capabilities, lower loss, as well as the possibility to engineer wide bandgaps at higher frequencies $[8]$.

Experimental setup. We have experimentally demonstrated the photonic-aided $\mathrm{THz}$-wave wired communication system over 1-m hollow-core fiber (HCF) in (Figure 1(a)) [9]. To approach the Shannon limit of the $\mathrm{THz}$ channel, the probabilistically shaped quadrature amplitude modulation (PS-QAM) signal is generated by using the probabilistic amplitude shaping (PAS) scheme based on low density parity check (LDPC) encoder with $25 \%$ overhead ( 0.8 code rate). A tunable external cavity laser (ECL1) with 100-kHz linewidth as the optical carrier is modulated by an $\mathrm{I} / \mathrm{Q}$ modulator with 30-GHz $3 \mathrm{~dB}$ bandwidth. Then, a cascaded erbium-doped fiber amplifier (EDFA) is used to amplify the modulated signals in order to compensate the insertion loss of the optics. Another laser ECL2 is applied as the optical local oscillator (LO), and the frequency space between the optical carrier ECL1 and optical LO ECL2 is $325 \mathrm{GHz}$. A polarizationmaintaining optical coupler (PM-OC) is used to couple the two lasers. Subsequently, the uni-travelling carrier photodiodes (UTC-PD) generate the $325 \mathrm{GHz} \mathrm{THz}$-wave signal and then transmit over 1-m THz HCF. Figure 1(a) shows the cross section of the HCF. In order to obtain flexibility, the hollow substrate of the THz HCF uses a polycarbonate

\footnotetext{
* Corresponding author (email: yujianjun@pmlabs.com.cn)
} 

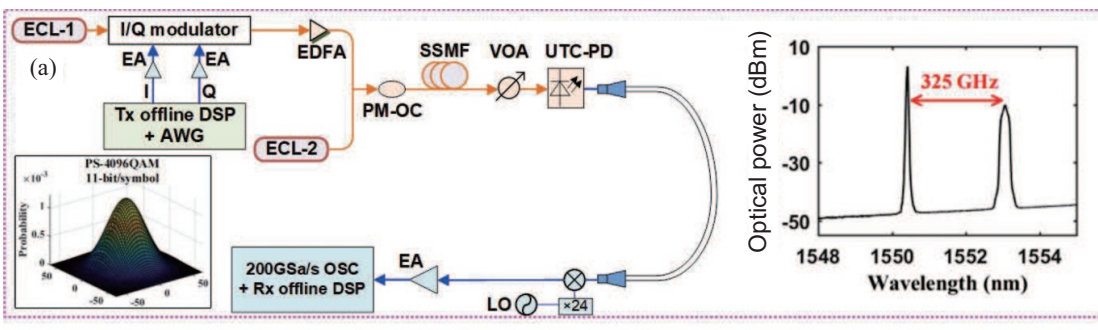

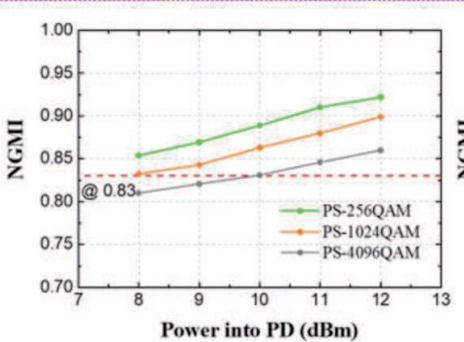

Figure 1 (Color online) (a) Experimental setup for 352-Gbit/s terahertz wired transmission over hollow-core fiber at 325 GHz; (b) NGMI of 32-Gbaud PS-256QAM, PS-1024QAM, and PS-4096QAM THz signals versus PD received power at BtB case and after 1-m HCF transmission, respectively. AWG, arbitrary waveform generator; DSP, digital signal processing; EA, electrical amplifier; SSMF, standard single mode fiber; VOA, variable optical attenuator; OSC, oscilloscope.

tube, which can be coiled and bent. The inner metal plating layer is formed from the silver film with $0.3 \mu \mathrm{m}$ thickness. The linear transmission loss of the THz $\mathrm{HCF}$ is $1.33 \mathrm{~dB} / \mathrm{m}$ at $300 \mathrm{GHz}$, and the inner diameter is $3.6 \mathrm{~mm}[10]$. At the receiver side, the received $\mathrm{THz}$ signals over $1-\mathrm{m} \mathrm{HCF}$ transmission are sampled by a real-time $200-\mathrm{GSa} / \mathrm{s}$ sampling rate oscilloscope with 59-GHz 3 dB bandwidth.

Results and discussion. The measured normalized generalized mutual information (NGMI) of 32-Gbaud PS256QAM, PS-1024QAM, and PS-4096QAM signals versus $\mathrm{PD}$ received power at back-to-back $(\mathrm{BtB})$ case and after 1$\mathrm{m}$ HCF transmission are shown in Figure 1(b), respectively. At BtB case, the NGMI of PS-256QAM and PS-1024QAM signals are all above the $0.83-\mathrm{NGMI}$ LDPC threshold. For PS-4096QAM signal, when the PD received power reaches $10 \mathrm{dBm}$, the post-FEC error free can be obtained. Moreover, there is around $0.5-\mathrm{dB}$ power penalty after $1-\mathrm{m}$ HCF transmission compared with the BtB case. For PS-4096QAM signal, the received power of the PD is improved to $10.5 \mathrm{dBm}$ at the 0.83-NGMI LDPC threshold. For PS-1024QAM signal, the required power into $\mathrm{PD}$ is $8.6 \mathrm{dBm}$ at 0.83 -NGMI threshold. By using 32-Gbaud PS-4096 QAM signal, the record-high 352 Gbps single line rate (275.2-Gbps net data rate) of the $\mathrm{THz}$ wired transmission and $8.6 \mathrm{bit} / \mathrm{s} / \mathrm{Hz}$ net spectrum efficiency is obtained at 0.83-NGMI threshold with $25 \%$ overhead. The deployed $\mathrm{THz} \mathrm{HCF}$ has a wide wavelength range from visible light to far infrared and even $\mathrm{THz}$ band. Furthermore, the loss of the cladding modes propagating through the HCF is near to air loss window. The promising results show $\mathrm{THz} \mathrm{HCF}$ paves the way for $6 \mathrm{G} \mathrm{THz}$ wireless and wired link seamless integration network, enabling in-depth coverage of $\mathrm{THz}$ signal in indoor scenarios.

\section{References}

1 Yang Y H, Yamagami Y, Yu X B, et al. Terahertz topological photonics for on-chip communication. Nat Photonics, 2020, 14: 446-451

2 Yu X B, Miyamoto T, Obata K, et al. Direct terahertz communications with wireless and fiber links. In: Proceedings of the 44th International Conference on Infrared, Millimeter, and Terahertz Waves (IRMMW-THz), 2019

3 Zhang L, Pang X D, Jia S, et al. Beyond $100 \mathrm{~Gb} / \mathrm{s}$ optoelectronic terahertz communications: key technologies and directions. IEEE Commun Mag, 2020, 58: 34-40

4 Nagatsuma T, Ducournau G, Renaud C C. Advances in terahertz communications accelerated by photonics. Nat Photon, 2016, 10: 371-379

5 Sengupta K, Nagatsuma T, Mittleman D M. Terahertz integrated electronic and hybrid electronic-photonic systems. Nat Electron, 2018, 1: 622-635

6 Horst Y, Blatter T, Kulmer L, et al. Transparent opticalTHz-optical Link transmission over $5 / 115 \mathrm{~m}$ at 240/190 Gbit/s enabled by plasmonics. In: Proceedings of Optical Fiber Communication Conference, San Diego, 2021

7 Yu J J, Li X Y, Tang X L, et al. High-speed signal transmission at W-band over dielectric-coated metallic hollow fiber. IEEE Trans Microwave Theor Techn, 2015, 63: 1836-1842

8 Ung B, Mazhorova A, Dupuis A, et al. Polymer microstructured optical fibers for terahertz wave guiding. Opt Express, 2011, 19: 848-861

9 Ding J J, Wang Y Y, Zhang J, et al. Demonstration of 352-Gbit/s single line rate PS-4096QAM THz wired transmission over hollow-core fiber. In: Proceedings of the 26th Optoelectronics and Communications Conference (OECC), Hong kong, 2021

10 He M H, Zeng J F, Zhang X, et al. Transmission and imaging characteristics of flexible gradually tapered waveguide at 03 THz. Opt Express, 2021, 29: 8430-8440 